\title{
Utilization of Advanced Computational Methods to Predict Spring-back of Alu- minium Alloys in Automotive Industry
}

Pavel Solfronk, Jiri Sobotka, David Korecek

Department of Engineering Technology, Technical University of Liberec. Studentská 1402/2,

46117 Liberec. Czech Republic. E-mail: pavel.solfronk@tul.cz, jiri.sobotka@tul.cz, david.korecek@tul.cz

The automotive industry is one of the most dynamically developing segments of the industrial production all over the World. The implementation of still more demanding emission limits for newly developed cars forces car producers to continuously reduce the fuel consumption of cars. As one possibility there are used hybrid drive units in combination with a redesigning the car-body while maintaining the highest possible level of vehicle safety. Because of these reasons, the automotive industry has been increasingly forced to implement and process low density (lightweight) alloys - including aluminium-based alloys. So these materials are subjected to high demands both in terms of mechanical properties and technological workability in the mass production process. The utilization of mathematical modelling (numerical simulations) at production processes is now one of the standards in all phases at designing and production of the car-body and allows implementation of variable shape modifications in a relatively short time scale. Moreover, it enable to detect the potential production problems as well. In this paper, the influence of the kinematic hardening model on the accuracy of spring-back prediction is shown in comparison with the commonly used isotropic hardening model. For deformation analysis, a simple workpiece having "U-shape" of EN AW 6111 material was used. Such aluminium alloys is used for production car-body panels in the automotive industry. Achieved accuracy of numerical simulation results is here evaluated by the comparison shape obtained by numerical simulations and shape of experimentally bended workpiece.

Keywords: Aluminium Alloys, Spring-back, Yield Criterion, Numerical Simulation, Bauschinger Effect

\section{Introduction}

Nowadays are on the sheet stampings posing quite strict requirements mainly in light of their strength, surface quality and dimensional accuracy. Stiffness and strength of produced part are essentially influenced by its shape and selection of the material. Design changes at the new types of car-bodies quite markedly increase the requirements for shape and dimensional accuracy of sheet stampings and it also forces the sheet processors to implement the newest methods which can fulfil these goals. Achieving the shape and dimensional accuracy of the formed part in bending is closely connected mainly with the material spring-back. Spring-back of the drawn stampings differs by its technological principle from products produced only by bending. Difference rests mainly in the deformation evolution and stress state on the drawing and bending edge of tool. At the conventional bending, direction of major stress in the cross-section area is both tensile (on the outer side) and compressive one (on the inner side). Quite important is fact that during bending process is changed just magnitude but not sense of these stresses. Thus there is not influence of the Bauschinger effect. However, at the drawing process is material in the first phase bended on the drawing edge and then it is straightened in the second phase. Thus here takes place Bauschinger effect during the material hardening. This paper is focused on the area of utilization mathematical modelling for spring-back prediction at sheet stampings. An anisotropic yield criterion termed as Vegter Lite in combination with isotropic and kinematic hardening was chosen to evaluate the influence of Bauschinger effect at prediction spring-back by means of mathematical modelling. This yield criterion Vegter Lite is one of the newest and most frequently used yield criterions in the automotive industry based on a complex performance of experimental measurements of tested material. The kinematic hardening model, known as Yoshida-Uemori, rests in utilization of cyclic test to define its parameters, because there occurs repeated transition to plastic state in tension and compression. In the field of sheet metal forming, this kinematic hardening is thus termed as YoshidaUemori by the top mathematical computation model and is still being developed and improved in collaboration with the world's leading universities and sheet metal manufacturers for the automotive industry. The aluminium alloy EN AW 6111 of 0.9 $\mathrm{mm}$ thickness, which is quite commonly used in automotive industry, was chosen to evaluate the 
influence of Bauschinger effect on the spring-back prediction with the help of mathematical modelling [1, $2,3]$.

\section{Methodological bases and experimental part}

In this chapter are described all tests which are needed to be performed to properly predict the magnitude of spring-back by the numerical simulation. Among them it can be found static tensile test, hydraulic bulge test and cyclic test.

\subsection{Static tensile test}

To define the Vegter Lite material model, there was needed to carry out the static tensile test and determined the normal anisotropy coefficients in the directions $0^{\circ}, 45^{\circ}$ and $90^{\circ}$ regarding the rolling direction. Tests conditions were chosen to be in accordance with the standards EN ISO 6892-1 and EN ISO 10113. From the measured values of static tensile test in the individual rolling directions were subsequently computed the stress-strain curves (hardening curves) as true stress $\sigma[\mathrm{MPa}]$ vs. true plastic strain $\varepsilon_{\mathrm{pl}}$ [-]. Regarding the mathematical definition of hardening curves in the isotropic model, there were made their approximations by the powerlaw function (see equation 1). Mechanical values of the tested material, fitting constants $\left(K, n, \varepsilon_{0}\right)$ from that power-law function and also the values of the normal anisotropy coefficients $\left(\mathrm{r}_{\alpha}\right)$ in dependence on the rolling direction are summarized in Tab. 1.

$$
\sigma=\mathrm{K}\left(\varepsilon_{\mathrm{pl}}+\varepsilon_{0}\right)^{n}
$$

Where:

$\mathrm{K}$ - strength coefficient[MPa], $\mathrm{n}$ - strain hardening exponent[1], $\varepsilon_{0}$ - offset of strain[1].

Tab. 1 Mechanical values, fitting constants and normal anisotropy coefficients in dependence on rolling direction

\begin{tabular}{|c|c|c|c|c|c|c|c|c|}
\hline $\begin{array}{c}\text { Rolling } \\
\text { direction }\end{array}$ & $\begin{array}{c}\text { Yield } \\
\text { strength } \\
{[\mathbf{M P a}]}\end{array}$ & $\begin{array}{c}\text { Ultimate } \\
\text { strength } \\
{[\mathbf{M P a}]}\end{array}$ & $\begin{array}{c}\text { Uniform } \\
\text { elongation } \\
{[\%]}\end{array}$ & $\begin{array}{c}\text { Total } \\
\text { elongation } \\
{[\%]}\end{array}$ & $\begin{array}{c}\mathbf{K} \\
{[\mathbf{M P a}]}\end{array}$ & $\begin{array}{c}\mathbf{n} \\
{[-]}\end{array}$ & $\begin{array}{c}\mathbf{s}_{0} \\
{[-]}\end{array}$ & $\begin{array}{c}\mathbf{r}_{\alpha} \\
{[-]}\end{array}$ \\
\hline $0^{\circ}$ & $141.8 \pm 0.47$ & $259.1 \pm 1.14$ & $21.3 \pm 0.21$ & $26.8 \pm 0.19$ & 492.51 & 0.2682 & 0.00533 & 0.7155 \\
\hline $45^{\circ}$ & $134.4 \pm 0.29$ & $251.6 \pm 0.98$ & $24.2 \pm 0.33$ & $25.7 \pm 0.37$ & 473.88 & 0.2686 & 0.00575 & 0.4631 \\
\hline $90^{\circ}$ & $133.0 \pm 0.58$ & $248.8 \pm 1.21$ & $23.2 \pm 0.42$ & $27.5 \pm 0.51$ & 473.48 & 0.2723 & 0.00548 & 0.6035 \\
\hline
\end{tabular}

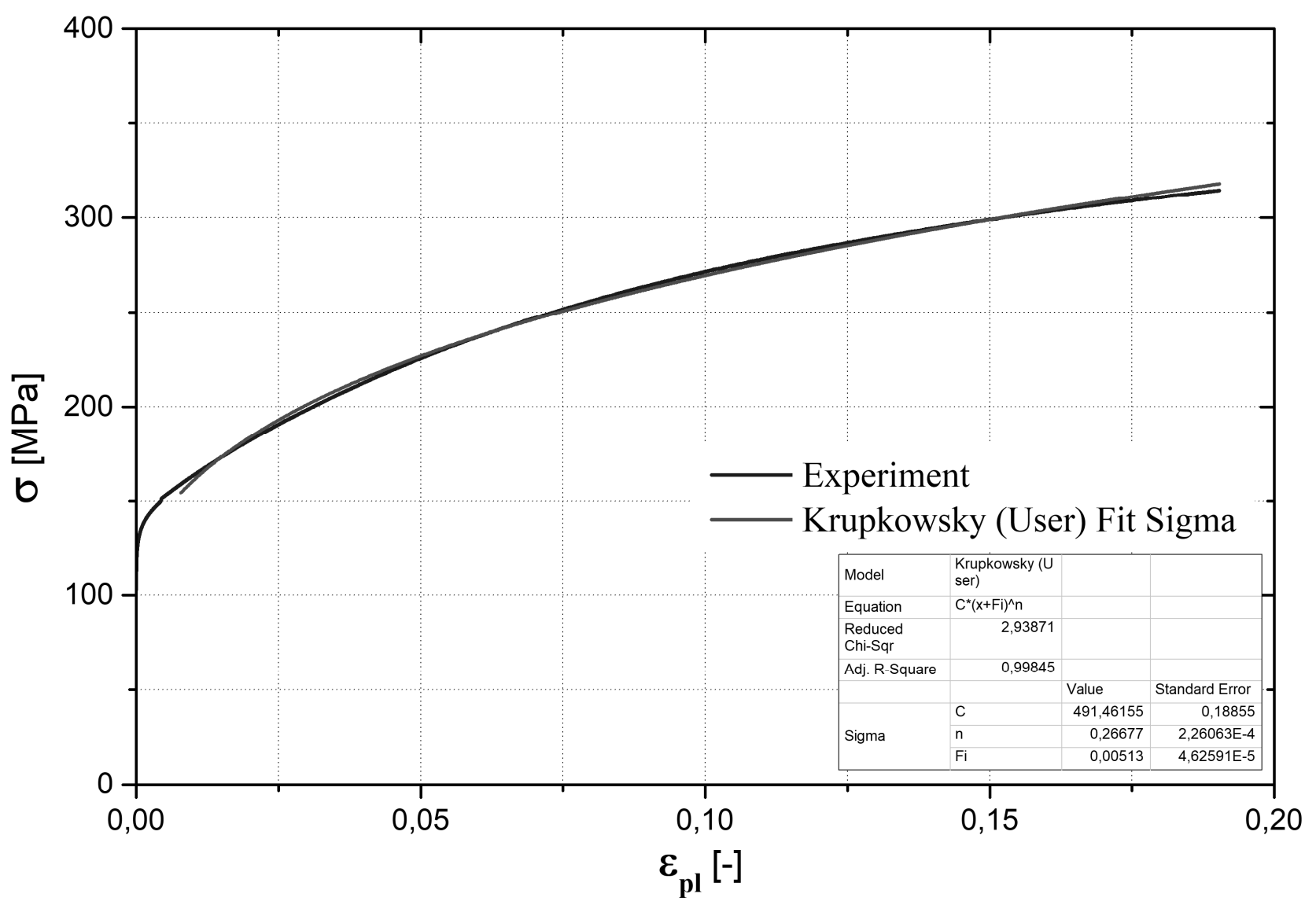

Fig. 1 Example of fitting measured values: static tensile test (stress-strain curve) 


\subsection{Hydraulic bulge test (HBT)}

For the precious definition of the Vegter yield criterion, there is especially very necessary to carry out so-called hydraulic bulge test (HBT). The hydraulic bulge test represented the second major part of the experiment. For this test is very important fact that there is a bi-axial stress state, because it is quite important "point" for the future utilization in the different yield criterions [4]. Due to the different stress state (comparing the static tensile test), there is necessary to compute so-called effective stress $\sigma_{\mathrm{EF}}$ $[\mathrm{MPa}]$ and effective strain $\varepsilon_{\mathrm{EF}}[-]$ for the relevant stress-strain curve. Values of fitting constants (equation 1) for the HBT were as following: $\mathrm{K}=505.41 \mathrm{MPa}, \mathrm{n}=0.30431$ and finally $\varepsilon_{0}=$ 0.003635 . Such values are very important, because from them is subsequently computed the significant bi-axial point in the advanced computational models in numerical simulations (e.g. at Vegter yield criterion). Beside values of uni-axial tensile (eventually compression) test and normal anisotropy coefficients, these quantities are crucial for the proper computation of required yield criterion (Vegter lite). Example of evaluation HBT is shown in Fig. 2.

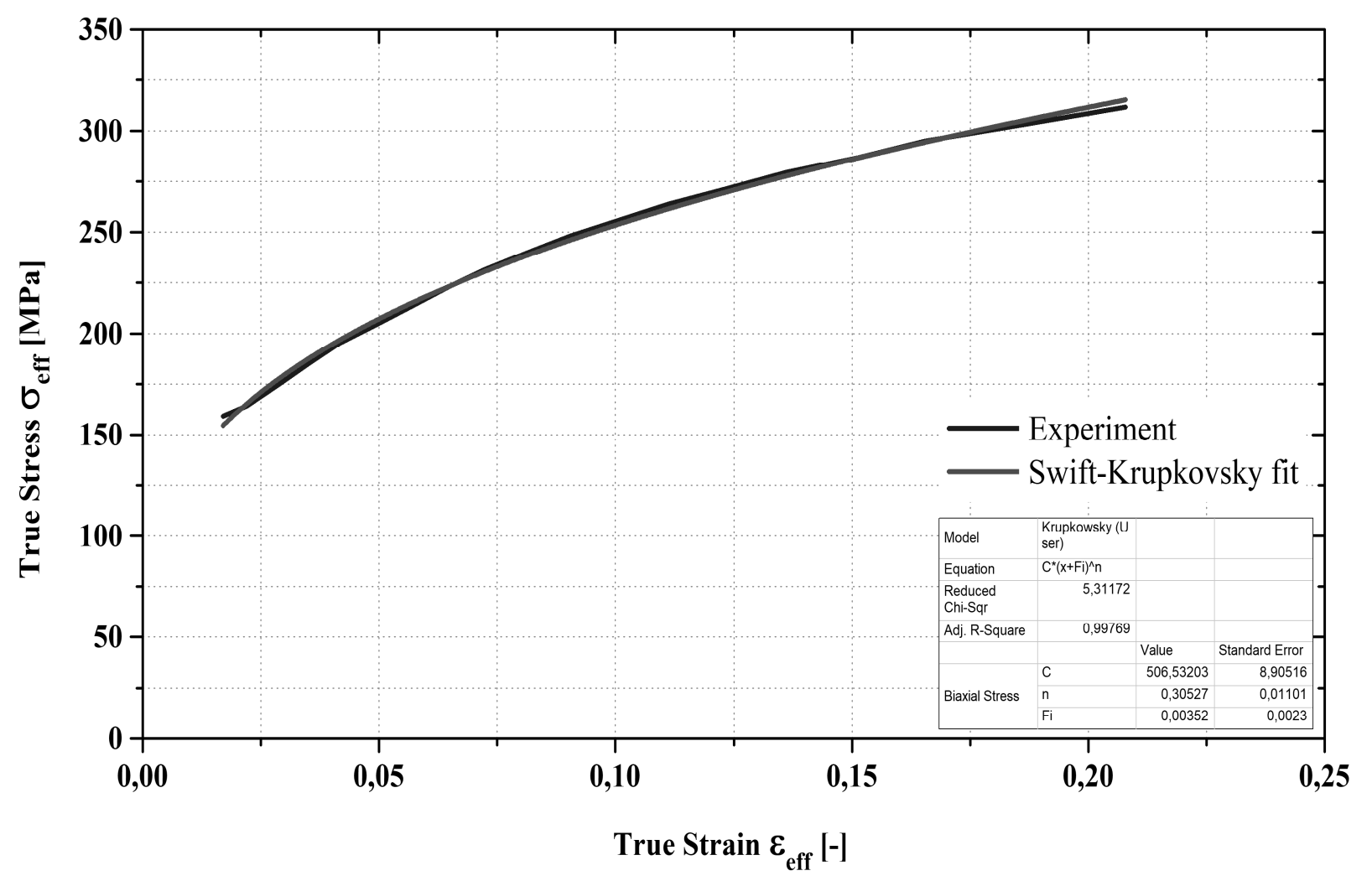

Fig. 2 Example of fitting measured values: bydraulic bulge test (stress-strain curve)

\subsection{Cyclic test}

In addition to the previous tests, cyclic test (where takes effect the change of loading direction $(+,-)$ in the tested sample) was also done to properly define the material model termed as Yoshida-Uemori [5, 6, 7]. Due to the compressive stress states, own performance of this test for sheet samples is quite demanding. Moreover, there is loss of stability resulting as sample buckling. Because of these reasons, at Department of Engineering Technology was designed the testing jig, which enables to carry out cyclic test on the testing machine TIRA Test 2300 that is primary designed for the static tensile test. So such testing jig was proposed as an additional jig for the clamping grips. Such testing jig consists of four subdivided supporting grips, which are hydraulically controlled and can prevent the sheet sample from buckling during the compression. Strain values are recorded by the contact length-gauge of high accuracy. As a result from this test, there is cyclically repeating course of tensile and compressive stress in dependence on deformation. The offset of the individual measured curves from monitored cycles rests in the magnitude of Bauschinger effect for tested material [8]. The whole arrangement of the cyclic test is shown in Fig. 3 (left). Results of the measured magnitudes are given in Fig. 3 (right). 

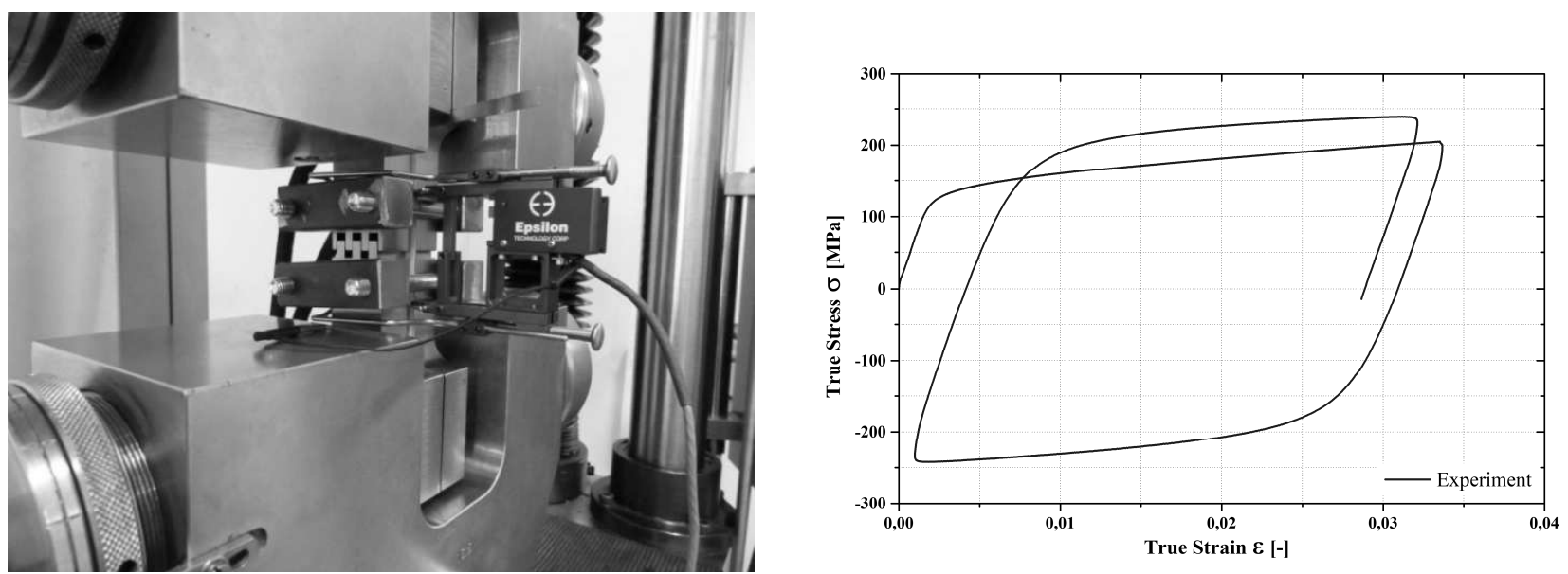

Fig. 3 Arrangement of the cyclic test on the device TIRA Test 2300 (left) and results of the cyclic test for aluminium alloy EN AW 6111 (right)

\subsection{Experimental measurement of the spring-back}

For the experimental determination of spring-back is suitable to choose such test when change of the stress state occurs in the bending area, because just in this case there can be fully developed so-called Bauschinger effect [4]. With respect to the equipment of KSP TUL laboratory, for the spring-back analysis was chosen test of bending workpiece (blank) into "U" shape in the tool with fixed blank-holder. This experiment simulates the process that occurs in a tool at sheet drawing. During the test, the workpiece is 2 times bended with the opposite bending direction. The principle of this test is evident from Fig. 4 (left), where is shown the own arrangement of bending tool. The test conditions were as following: distance between fixed blank-holder and die was $1 \mathrm{~mm}$ (i.e. draw clearance between tool and workpiece was 0,1

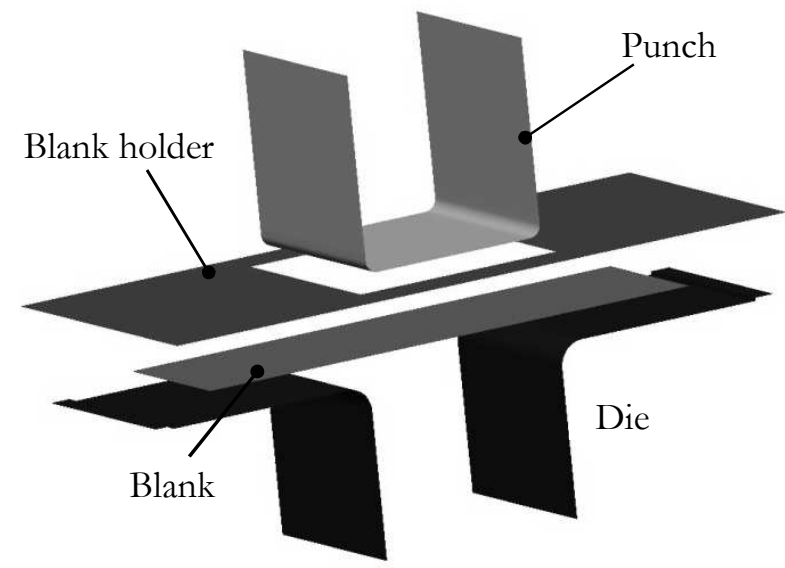

$\mathrm{mm}$ ), die drawing radius was $\mathrm{R} 5$, punch drawing radius was R2.5, workpiece dimensions (rectangular shape) were as $30 \mathrm{~mm} \times 110 \mathrm{~mm}$, bending direction was selected as perpendicular to the sheet rolling direction and own bending was performed on the LENP 40 eccentric press. After finishing such bending test, specimen was measured by the dimensional and shape analysis on the 3D coordinate measuring device SOMET XZY 464 equipped with the relevant software TANGO1! 3D. As a results of the experimental measurement there are coordinates of 40 points in *.step format, which represents the actual shape of sample. Principle of measuring the real shape is shown in Fig. 4. Obtained shape of the bended blank was subsequently used as a comparative criterion to verify the match between results of real experiment and numerical simulation (in this case was used software PAM-STAMP 2G).

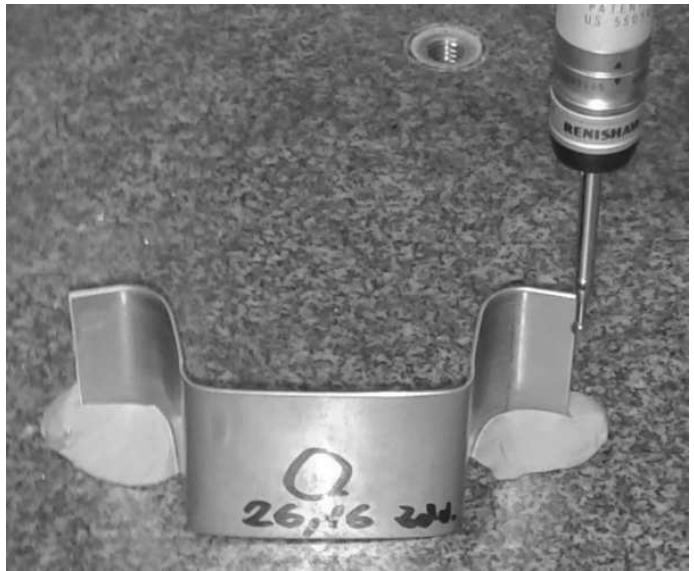

Fig. 4 Principle of bending test (left) and measurement on the 3D coordinate measuring device (right)

\section{Numerical simulation of the spring-back}

Software PAM-STAMP $2 \mathrm{G}$ was used for numerical simulation of the spring-back analysis. For its mathematical computation was applied yield criterion termed as Vegter Lite and such yield criterion was combined always both with the isotropic and kinematic hardening model (so-called YoshidaUemori model). That's why there were used two computational models and results compared with that ones from the real experiments. 


\subsection{Definition of the material models}

For the numerical simulation, the yield criterion known as Vegter Lite was chosen. It allows defining different yield strengths in dependence on the rolling direction just from the static tensile test. In addition to that, there is also possible to use results of the experimental tests performed under different stress states. Most frequently, a bi-axial test (even. shear test) is performed to improve accuracy of the yield criterion. To define these yield criterion conditions, there was necessary into the material card enter the following quantities: Young's modulus, Poisson's ration, density of material, normal anisotropic coefficients and yield strength magnitudes in directions $0^{\circ}, 45^{\circ}$ and $90^{\circ}$. The yield criterion Vegter Lite is further combined with the isotropic hardening of material defined by the hardening curve according to equation (1) and the kinematic hardening of the material termed as Yoshida Uemori. The MatPara software was used to define parameters defining the kinematic hardening model, whose input data are taken as results from the cyclic test in combination with the static tensile test. Material cards in the software PAM-STAMP 2G environment for both tested computational models are shown in Fig. 5.

The boundary conditions of the numerical simulation were set in accordance with the performed experiment. Thus as a drawing depth it was taken 25 mm without using the lower blank-holder.
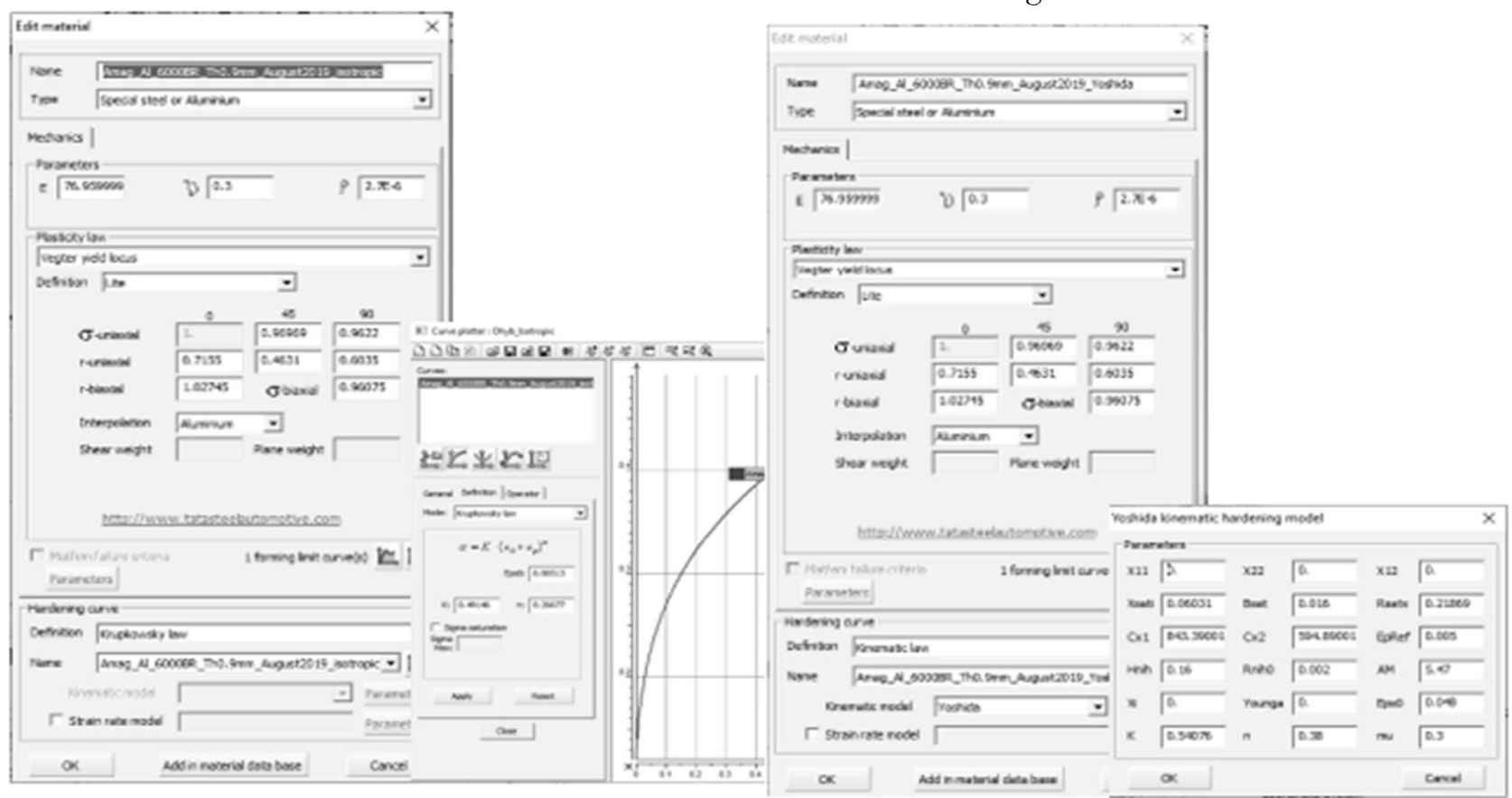

Fig. 5 Material cards: Vegter Lite + isotropic hardening (left) and Vegter Lite + kinematic hardening Yoshida-Uemori (right)

\subsection{Comparison of results from numerical simulations and real experiments}

Accuracy of the computational model was tested based upon the shape matching between the results of numerical simulation and "U-bending" of workpiece.
In Fig. 6 are graphically shown contours of sheets obtained by means of numerical simulation and contour measured from the performed experiment (real experiment).

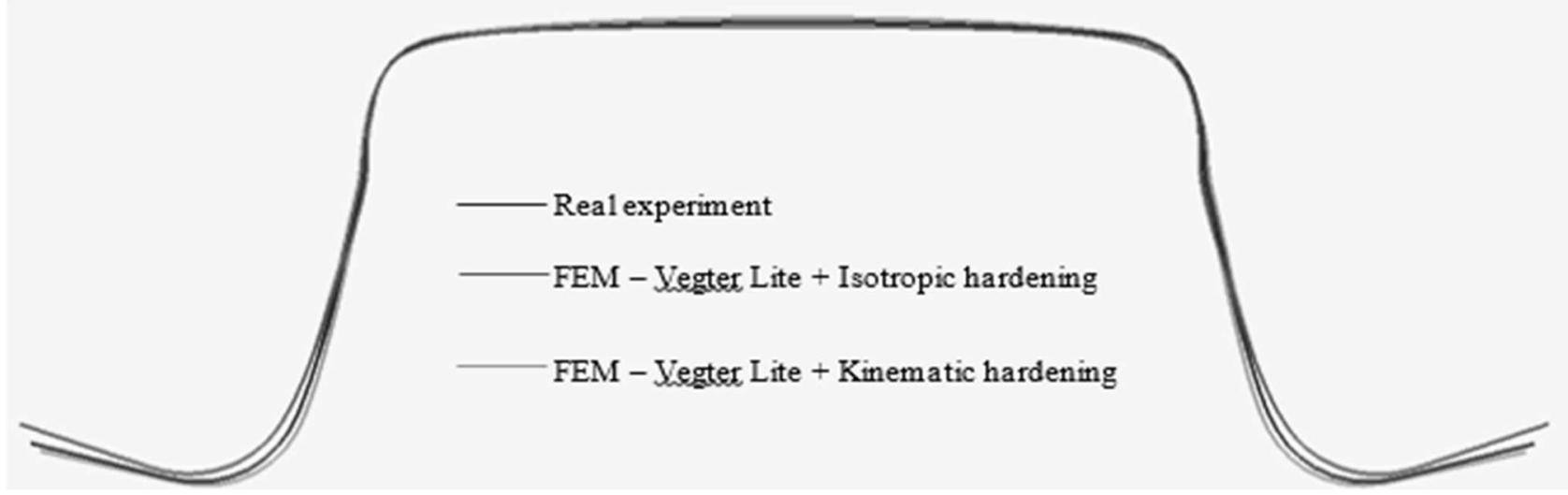

Fig. 6 Final comparison of results: shape contours for different tests 


\section{Conclusion}

From the performed experiments and numerical simulations is evident that utilization of the kinematic hardening model in the mathematical modelling of the thin sheets drawing process leads to a significant accuracy improvement of results for the spring-back prediction. This is particularly important with respect to continuing thickness reduction of materials used in the car-body design. In the experimental part, the stress state from tensile to compressive one was changed only 2 times. However, in the real stamping process, this change occurs repeatedly several times on the drawing beads, drawing edge, offsets and so on. For this reason, the Bauschinger effect in the real stamping process is more intensive than in the proposed experiment. On this basis, it can be reasonably assumed that utilization of the kinematic hardening models for real stampings will lead to a qualitatively higher level of numerical simulation in light of its deformation distribution and spring-back prediction on the stamping. Thus there can be stated that for more complex stampings, where it is quite difficult to predict the effect of the new technological methodologies applied in the stamping process, the time-consuming acquisition of the input data for kinematic models can be offset by savings in the production preparation process.

\section{Acknowledgement}

This publication was written at the Technical University of Liberec as part of the project SGS 21280 "Research and development for innovation of materials and production technologies with application potential in mechanical engineering" with the support of the Specific University Research Grant, as provided by the Ministry of Education, Youth and Sports of the Czech Republic in the year 2019.

\section{References}

[1] PUCHNIN, M. et al. (2016) Comparison of ABI Technique and Standard Methods in
Measuring Mechanical Properties of Aluminium Al-alloys. pp. 600-607. Manufacturing Technology, Volume 16.

[2] CHUNJIAN, M., XUETAO, W. (2014) Springback Research of V-type Sheet Metal forming based on the Adjustable Drawbead and Variable Blank-holder Force Cooperative Control Technology. pp. 618-625. Manufacturing Tecbnology, Volume 14.

[3] Chunjian, M., XUETAO, W. (2014) Study on Springback Rule of AZ31B Magnesium Alloy Axisymmetric Part's Warm Single Point Incremental Forming without Mould. pp. 626632. Manufacturing Technology, Volume 14.

[4] ASM HANDBOOK. (2000) Volume 8 - Mechanical Testing and Evaluation. pp. 104-130.ASM International. Materials Park.

[5] HASSAN, H. U. et al. (2016) Accurate springback prediction in deep drawing using prestrain based multiple cyclic stress-strain curves in finite element simulation. pp 229-241. International Journal of Mechanical Sciences

[6] SHUN-LAI, Z. et al. (2013) Evaluating the significance of hardening behavior and unloading modulus under strain reversal in sheet springback prediction. pp. 194-204 International Journal of Mechanical Sciences.

[7] TAHERIZADEH, A. et al. (2009) Finite element simulation of springback for a channel draw process with drawbead using different hardening models. pp. 314-325. International Journal of Mechanical Sciences.

[8] YOSHIDA, F., UEMORI, T. (2003) A model of large-strain cyclic plasticity and its application to springback simulation. pp. 1687-1702. International Journal of Mechanical Sciences. 